PROCEEDINGS OF THE

AMERICAN MATHEMATICAL SOCIETY

Volume 131, Number 1, Pages 291-302

S 0002-9939(02)06557-7

Article electronically published on May 8, 2002

\title{
ASYMPTOTICS OF ALMOST HOLOMORPHIC SECTIONS OF AMPLE LINE BUNDLES ON SYMPLECTIC MANIFOLDS: AN ADDENDUM
}

\author{
BERNARD SHIFFMAN AND STEVE ZELDITCH \\ (Communicated by Christopher D. Sogge)
}

\begin{abstract}
We define a Gaussian measure on the space $H_{J}^{0}\left(M, L^{N}\right)$ of almost holomorphic sections of powers of an ample line bundle $L$ over a symplectic manifold $(M, \omega)$, and calculate the joint probability densities of sections taking prescribed values and covariant derivatives at a finite number of points. We prove that they have a universal scaling limit as $N \rightarrow \infty$. This result will be used in another paper to extend our previous work on universality of scaling limits of correlations between zeros to the almost-holomorphic setting.
\end{abstract}

\section{INTRODUCTION}

This note is an addendum to our study in $[\mathrm{ShZe} 2$ of almost holomorphic sections of powers of ample line bundles $L^{N} \rightarrow M$ over almost complex symplectic manifolds $(M, \omega, J)$. Motivated by the important role now played by 'asymptotically holomorphic' sections in symplectic geometry (see Aur, Don and many related articles), we studied in [ShZe2] a conceptually related but different class $H_{J}^{0}\left(M, L^{N}\right)$ of 'almost holomorphic' sections defined by a method of Boutet de Monvel and Guillemin $\mathrm{BoGu}]$. The main results of [ShZe2] were the scaling limit law of the almost-complex Szegö projectors

$$
\Pi_{N}: L^{2}\left(M, L^{N}\right) \rightarrow H_{J}^{0}\left(M, L^{N}\right)
$$

and various geometric consequences of it.

Our purpose in this addendum is to develop [ShZe2] in a probabilistic direction, in the spirit of our earlier work with P. Bleher on the holomorphic case [BSZ1, and to complete the discussion in BSZ2] of correlations between zeros in the symplectic case. The space $H_{J}^{0}\left(M, L^{N}\right)$ is finite dimensional and carries a natural Hermitian inner product (see (7) and (14)). We endow the unit sphere $S H_{J}^{0}\left(M, L^{N}\right)$ in $H_{J}^{0}\left(M, L^{N}\right)$ with Haar probability measure $\nu_{N}$ and consider the joint probability distribution (JPD)

$$
\mathbf{D}_{\left(z^{1}, \ldots, z^{n}\right)}^{N}=D^{N}\left(x^{1}, \ldots, x^{n}, \xi^{1}, \ldots, \xi^{n} ; z^{1}, \ldots, z^{n}\right) d x d \xi
$$

of a random section $s_{N} \in S H^{0}\left(M, L^{N}\right)$ having the prescribed values $x^{1}, \ldots, x^{n}$ and derivatives $\xi^{1}, \ldots, \xi^{n}$ at $n$ points $z^{1}, \ldots, z^{n} \in M$. To be more precise, we choose

Received by the editors August 3, 2001.

2000 Mathematics Subject Classification. Primary 53D50, 53D35, 60 D05.

Research partially supported by NSF grants \#DMS-9800479, \#DMS-0100474 (first author) and \#DMS-0071358 (second author). 
local complex coordinates $\left\{z_{1}, \ldots, z_{m}\right\}$ and a nonvanishing local section $e_{L}$ of $L$ on an open set containing the points $\left\{z^{1}, \ldots, z^{n}\right\}$; then $\mathbf{D}_{\left(z^{1}, \ldots, z^{n}\right)}^{N}$ is the JPD of the $n(2 m+1)$ complex random variables

$$
\begin{gathered}
x^{p}=\left.\left\langle e_{L}^{* N}, s_{N}\right\rangle\right|_{z^{p}}, \xi_{q}^{p}=\left.\left\langle e_{L}^{* N}, N^{-\frac{1}{2}} \nabla_{\partial / \partial z_{q}} s_{N}\right\rangle\right|_{z^{p}}, \\
\xi_{m+q}^{p}=\left.\left\langle e_{L}^{* N}, N^{-\frac{1}{2}} \nabla_{\partial / \partial \bar{z}_{q}} s_{N}\right\rangle\right|_{z^{p}}, \quad 1 \leq p \leq n, 1 \leq q \leq m .
\end{gathered}
$$

As the name implies, almost holomorphic sections behave in the large $N$ limit much as do holomorphic sections on complex manifolds. The main result of this note bears this out by showing that the JPD in the almost complex symplectic case has the same universal scaling law as in the holomorphic case, thereby finishing the proof of Theorem 1.2 of BSZ2] on universal scaling limits of correlations between zeros in the symplectic case.

Theorem 1.1. Let $L$ be the complex line bundle over a $2 m$-dimensional compact integral symplectic manifold $(M, \omega)$ with curvature $\omega$. Let $P_{0} \in M$, and choose a local frame e for $L$ and local complex coordinates centered at $P_{0}$ so that $\left.\omega\right|_{P_{0}}$ and $\left.g\right|_{P_{0}}$ are the usual Euclidean Kähler form and metric respectively, $\left\|e_{L}\right\|_{P_{0}}=1$, and $\left.\nabla e_{L}\right|_{P_{0}}=0$. Then

$$
\mathbf{D}_{\left(z^{1} / \sqrt{N}, \ldots, z^{n} / \sqrt{N}\right)}^{N} \longrightarrow \mathbf{D}_{\left(z^{1}, \ldots, z^{n}\right)}^{\infty} \quad\left(\left(z^{1}, \ldots, z^{n}\right) \in \mathbb{C}^{m n}\right)
$$

where the measures $\mathbf{D}_{\left(z^{1}, \ldots, z^{n}\right)}^{\infty}=\gamma_{\Delta^{\infty}(z)}$ are the same universal Gaussian measures as in the holomorphic case; in particular, they are supported on the holomorphic 1 -jets.

Let us review the formula for $\gamma_{\Delta^{\infty}(z)}$. We recall that a (complex) Gaussian measure on $\mathbb{C}^{k}$ is a measure of the form

$$
\gamma_{\Delta}=\frac{e^{-\left\langle\Delta^{-1} z, \bar{z}\right\rangle}}{\pi^{k} \operatorname{det} \Delta} d z
$$

where $d z$ denotes Lebesgue measure on $\mathbb{C}^{k}$, and $\Delta$ is a positive definite Hermitian $k \times k$ matrix. The matrix $\Delta=\left(\Delta_{\alpha \beta}\right)$ is the covariance matrix of $\gamma_{\Delta}$ :

$$
\left\langle z_{\alpha} \bar{z}_{\beta}\right\rangle_{\gamma_{\Delta}}=\Delta_{\alpha \beta}, \quad 1 \leq \alpha, \beta \leq k
$$

Since the universal limit measures $\gamma_{\Delta \infty}(z)$ vanish along non-holomorphic directions, they are singular measures on the space of all 1-jets. To deal with singular measures, we introduce in 93 generalized Gaussian measures whose covariance matrices (3) are only semi-positive definite; a generalized Gaussian measure is simply a Gaussian measure supported on the subspace corresponding to the positive eigenvalues of the covariance matrix.

The covariance matrix $\Delta^{\infty}(z)$ is given along holomorphic directions by the same formula as in the holomorphic case [BSZ1, (97)], namely

$$
\Delta^{\infty}(z)=\frac{m !}{c_{1}(L)^{m}}\left(\begin{array}{cc}
A^{\infty}(z) & B^{\infty}(z) \\
B^{\infty}(z)^{*} & C^{\infty}(z)
\end{array}\right)
$$


where $z=\left(z^{1}, \ldots, z^{n}\right)$ and

$$
\begin{aligned}
A^{\infty}(z)_{p^{\prime}}^{p} & =\Pi_{1}^{\mathbf{H}}\left(z^{p}, 0 ; z^{p^{\prime}}, 0\right), \quad \Pi_{1}^{\mathbf{H}}(u, 0 ; v, 0)=\frac{1}{\pi^{m}} e^{u \cdot \bar{v}-\frac{1}{2}\left(|u|^{2}+|v|^{2}\right),}, \\
B^{\infty}(z)_{p^{\prime} q^{\prime}}^{p} & = \begin{cases}\left(z_{q^{\prime}}^{p}-z_{q^{\prime}}^{p^{\prime}}\right) \Pi_{1}^{\mathbf{H}}\left(z^{p}, 0 ; z^{p^{\prime}}, 0\right) & \text { for } 1 \leq q \leq m, \\
0 & \text { for } m+1 \leq q \leq 2 m,\end{cases} \\
C^{\infty}(z)_{p^{\prime} q^{\prime}}^{p q} & = \begin{cases}\left(\delta_{q q^{\prime}}+\left(\bar{z}_{q}^{p^{\prime}}-\bar{z}_{q}^{p}\right)\left(z_{q^{\prime}}^{p}-z_{q^{\prime}}^{p^{\prime}}\right)\right) \Pi_{1}^{\mathbf{H}}\left(z^{p}, 0 ; z^{p^{\prime}}, 0\right) \\
\text { for } 1 \leq q, q^{\prime} \leq m, \\
0 & \text { for } \max \left(q, q^{\prime}\right) \geq m+1 .\end{cases}
\end{aligned}
$$

In other words, the coefficients of $\Delta^{\infty}(z)$ corresponding to the anti-holomorphic directions vanish, while the coefficients corresponding to the holomorphic directions are given by the Szegö kernel $\Pi_{1}^{\mathbf{H}}$ for the reduced Heisenberg group (see BBSZ1. $\S 1.3 .2]$ ) and its covariant derivatives.

A technically interesting novelty in the proof is the role of the $\bar{\partial}$ operator. In the holomorphic case, $\mathbf{D}_{\left(z^{1}, \ldots, z^{n}\right)}^{N}$ is supported on the subspace of jets of sections satisfying $\bar{\partial} s=0$. In the almost complex case, sections do not satisfy this equation, so $\mathbf{D}_{\left(z^{1}, \ldots, z^{n}\right)}^{N}$ is a measure on the higher-dimensional space of all 1-jets. However, Theorem 1.1 says that the mass in the non-holomorphic directions shrinks to zero as $N \rightarrow \infty$.

An alternate statement of Theorem 1.1 involves equipping $H_{J}^{0}\left(M, L^{N}\right)$ with a Gaussian measure, and letting $\widetilde{\mathbf{D}}_{\left(z^{1}, \ldots, z^{n}\right)}^{N}$ be the corresponding joint probability distribution, which is a Gaussian measure on the complex vector space of 1-jets of sections. We show (Theorem 4.1) that these Gaussian measures $\widetilde{\mathbf{D}}^{N}$ also have the same scaling limit $\mathbf{D}^{\infty}$, so that asymptotically the probabilities are the same as in the holomorphic case, where universality was established in BSZ2. It is then easy to see that $\widetilde{\mathbf{D}}_{\left(z^{1}, \ldots, z^{n}\right)}^{N}=\gamma_{\Delta^{N}}$, where $\Delta^{N}$ is the covariance matrix of the random variables in (1). The main step in the proof is to show that the covariance matrices $\Delta^{N}$ underlying $\widetilde{\mathbf{D}}^{N}$ tend in the scaling limit to a semi-positive matrix $\Delta^{\infty}$. It follows that the scaled distributions $\widetilde{\mathbf{D}}^{N}$ tend to the generalized Gaussian $\gamma_{\Delta}$ 'vanishing in the $\bar{\partial}$-directions.'

In a subsequent article [ShZe3], we obtain further probabilistic results on holomorphic and almost holomorphic sections. Regarding almost holomorphic sections, we prove that a sequence $\left\{s_{N}\right\}$ of almost holomorphic sections satifies the bounds

$$
\left\|s_{N}\right\|_{\infty} /\left\|s_{N}\right\|_{L^{2}}=O(\sqrt{\log N}), \quad\left\|\bar{\partial} s_{N}\right\|_{\infty} /\left\|s_{N}\right\|_{L^{2}}=O(\sqrt{\log N})
$$

almost surely. Hence almost holomorphic sections satisfy bounds similar to those satisfied by asymptotically holomorphic sections in Don, Aur.

Finally, we mention some intriguing questions relating our probabalistic approach to almost holomorphic sections to the now-standard complexity-theoretic approach to asymptotically holomorphic sections in symplectic geometry, due to Donaldson and further developed by Auroux and others. From an analytical viewpoint (which of course is just one technical side of their work), the key results are existence theorems for one or several asymptotically holomorphic sections satisfying quantitative transversality conditions, such as

$$
s(z)=0 \Longrightarrow \| \bar{\partial} s(z)|<| \partial s(z) \mid \quad \forall z \in M
$$


in the case of one section. Can one use the probabalistic method to prove such existence results? It is the global nature of the problem which makes it difficult. On small balls, our methods rather easily give lower bounds for quantitative transversality of the type

$$
\mu\left\{s:|\bar{\partial} s(z)|<|\partial s(z)| \quad \forall z \in B_{\frac{D}{\sqrt{N}}}\left(z_{0}\right) \text { such that } s(z)=0\right\}>1-\frac{C_{\varepsilon}}{N^{1-\varepsilon}} .
$$

However, there are roughly $C_{m} N^{m}$ balls of radius $1 / \sqrt{N}$, so one cannot simply sum this small-ball estimate. To globalize, one would need to partition $M$ into small cubes as in Don and then analyze the dependence of transversality conditions from one cube to another.

Remark. This paper and [ShZe2] were originally contained in the archived preprint ShZe1. In revising that paper for publication as [ShZe2], we expanded the section on the Szegö kernel, added a discussion on transversality, and relocated the material on the JPD to this article. In particular, Theorem 1.1 is Theorem 0.2 of [ShZe1], and Theorem 4.1] is Theorem 5.4 of [ShZe1].

\section{BACKGROUND}

To avoid duplication, we only set up some basic notation and background, and refer to BSZ1, BSZ2, ShZe2 for further discussion and details.

Let $(M, \omega, J)$ denote a compact almost-complex symplectic manifold such that $\left[\frac{1}{\pi} \omega\right]$ is an integral cohomology class, and such that $\omega(J v, J w)=\omega(v, w)$ and $\omega(v, J v)>0$. We further let $(L, h, \nabla) \rightarrow M$ denote a quantizing Hermitian line bundle and metric connection with curvature $\frac{i}{2} \Theta_{L}=\omega$. We denote by $L^{N}$ the $N^{\text {th }}$ tensor power of $L$.

We give $M$ the Riemannian metric $g(v, w)=\omega(v, J w)$. We denote by $T^{1,0} M$, resp. $T^{0,1} M$, the holomorphic, resp. anti-holomorphic, sub-bundle of the complex tangent bundle $T M$; i.e., $J=i$ on $T^{1,0} M$ and $J=-i$ on $T^{0,1} M$.

We now recall our notion of 'preferred coordinates' from [ShZe2]. They are important because the universal scaling laws are only valid in such coordinates. A coordinate system $\left(z_{1}, \ldots, z_{m}\right)$ on a neighborhood $U$ of a point $P_{0} \in M$ is preferred at $P_{0}$ if any two of the following conditions (and hence all three) are satisfied:

i) $\partial /\left.\partial z_{j}\right|_{P_{0}} \in T^{1,0}(M)$, for $1 \leq j \leq m$,

ii) $\omega\left(P_{0}\right)=\omega_{0}$,

iii) $g\left(P_{0}\right)=g_{0}$,

where $\omega_{0}$ is the standard symplectic form and $g_{0}$ is the Euclidean metric:

$$
\begin{aligned}
& \omega_{0}=\frac{i}{2} \sum_{j=1}^{m} d z_{j} \wedge d \bar{z}_{j}=\sum_{j=1}^{m}\left(d x_{j} \otimes d y_{j}-d y_{j} \otimes d x_{j}\right), \\
& g_{0}=\sum_{j=1}^{m}\left(d x_{j} \otimes d x_{j}+d y_{j} \otimes d y_{j}\right) .
\end{aligned}
$$

Here we write $z_{j}=x_{j}+i y_{j}$, and we let $\left\{\frac{\partial}{\partial z_{j}}, \frac{\partial}{\partial \bar{z}_{j}}\right\}$ denote the dual frame to $\left\{d z_{j}, d \bar{z}_{j}\right\}$.

As in [BSZ1, BSZ2, ShZe2], it is advantageous to work on the associated principal $S^{1}$ bundle $X \rightarrow M$, and our Szegö kernels will be defined there. Let $\pi: L^{*} \rightarrow M$ denote the dual line bundle to $L$ with dual metric $h^{*}$, and put $X=\left\{v \in L^{*}\right.$ : 
$\left.\|v\|_{h^{*}}=1\right\}$. We let $r_{\theta} x=e^{i \theta} x(x \in X)$ denote the $S^{1}$ action on $X$. We then identify sections $s_{N}$ of $L^{N}$ with equivariant function $\hat{s}$ on $L^{*}$ by the rule

$$
\hat{s}_{N}(\lambda)=\left(\lambda^{\otimes N}, s_{N}(z)\right), \quad \lambda \in X_{z},
$$

where $\lambda^{\otimes N}=\lambda \otimes \cdots \otimes \lambda$; then $\hat{s}_{N}\left(r_{\theta} x\right)=e^{i N \theta} \hat{s}_{N}(x)$. We denote by $\mathcal{L}_{N}^{2}(X)$ the space of such equivariant functions transforming by the $N^{\text {th }}$ character.

When working on $X$, covariant derivatives on sections of $L$ become horizontal derivatives of equivariant functions. We consider preferred coordinates $\left(z_{1}, \ldots, z_{m}\right)$ centered at a point $P_{0} \in M$ and a local frame $e_{L}$ for $L$ such that $\left\|e_{L}\right\|_{P_{0}}=1$ and $\left.\nabla e_{L}\right|_{P_{0}}=0$. This gives us coordinates $\left(z_{1}, \ldots, z_{m}, \theta\right)$ on $X$ corresponding to $x=e^{i \theta}\left\|e_{L}(z)\right\| e_{L}^{*}(z) \in X$. We showed in [ShZe2, $\left.\S 1.2\right]$ that

$$
\frac{\partial^{h}}{\partial z_{j}}=\frac{\partial}{\partial z_{j}}+\left[\frac{i}{2} \bar{z}_{j}+O\left(|z|^{2}\right)\right] \frac{\partial}{\partial \theta}, \quad \frac{\partial^{h}}{\partial \bar{z}_{j}}=\frac{\partial}{\partial \bar{z}_{j}}-\left[\frac{i}{2} z_{j}+O\left(|z|^{2}\right)\right] \frac{\partial}{\partial \theta},
$$

where $\frac{\partial^{h}}{\partial z_{j}}$ (resp. $\frac{\partial^{h}}{\partial \bar{z}_{j}}$ ) denotes the horizontal lift of $\frac{\partial}{\partial z_{j}}$ (resp. $\frac{\partial}{\partial \bar{z}_{j}}$ ).

The almost-complex Cauchy-Riemann operator $\bar{\partial}_{b}$ on $X$ does not satisfy $\bar{\partial}_{b}^{2}=0$ in general, and usually it has no kernel. Following a method of Boutet de Monvel and Guillemin, we defined in ShZe2 the space $\mathcal{H}_{J}^{N}$ of equivariant almost-CR functions on $X$ as the kernel of a certain deformation $\bar{D}_{0}$ of the $\bar{\partial}_{b}$ operator on $\mathcal{L}_{N}^{2}(X)$. The space $H_{J}^{0}\left(M, L^{N}\right)$ is then the corresponding space of sections. The Szegö kernel $\Pi_{N}(x, y)$ is the kernel of the orthogonal projection $\Pi_{N}: \mathcal{L}_{N}^{2}(X) \rightarrow \mathcal{H}_{J}^{N}$. The dimension $d_{N}=\operatorname{dim} H_{J}^{0}\left(M, L^{N}\right)$ satisfies the Riemann-Roch formula (see [BoGu])

$$
d_{N}=\frac{c_{1}(L)^{m}}{m !} N^{m}+O\left(N^{m-1}\right) .
$$

Since $H_{J}^{0}\left(M, L^{N}\right)$ is finite dimensional, the Szegö kernel $\Pi_{N}$ is smooth and is given by

$$
\Pi_{N}(x, y)=\sum_{j=1}^{d_{N}} S_{j}^{N}(x) \overline{S_{j}^{N}(y)},
$$

where $\left\{S_{j}^{N}\right\}$ is an orthonormal basis for $H_{J}^{0}\left(M, L^{N}\right)$.

It would take us too far afield to discuss the definition or significance of the spaces $H_{J}^{0}\left(M, L^{N}\right)$ here; we refer the reader to [ShZe2] for background.

\section{A generalized Poincaré-Borel lemma}

In this section, we give a generalization of the Poincaré-Borel lemma concerning the asymptotics of linear push-forwards of measures on spheres of growing dimensions, which we shall use in the proof of Theorem 1.1

Recall that a Gaussian measure on $\mathbb{R}^{n}$ is a measure of the form

$$
\gamma_{\Delta}=\frac{e^{-\frac{1}{2}\left\langle\Delta^{-1} x, x\right\rangle}}{(2 \pi)^{n / 2} \sqrt{\operatorname{det} \Delta}} d x_{1} \cdots d x_{n},
$$

where $\Delta$ is a positive definite symmetric $n \times n$ matrix. The matrix $\Delta$ gives the second moments of $\gamma_{\Delta}$ :

$$
\left\langle x_{j} x_{k}\right\rangle_{\gamma_{\Delta}}=\Delta_{j k} .
$$

This Gaussian measure is also characterized by its Fourier transform

$$
\widehat{\gamma_{\Delta}}\left(t_{1}, \ldots, t_{n}\right)=e^{-\frac{1}{2} \sum \Delta_{j k} t_{j} t_{k}}
$$


If we let $\Delta$ be the $n \times n$ identity matrix, we obtain the standard Gaussian measure on $\mathbb{R}^{n}$,

$$
\gamma_{n}:=\frac{1}{(2 \pi)^{n / 2}} e^{-\frac{1}{2}|x|^{2}} d x_{1} \cdots d x_{n}
$$

with the property that the $x_{j}$ are independent Gaussian variables with mean 0 and variance 1 .

By a generalized complex Gaussian measure on $\mathbb{C}^{n}$, we mean a generalized Gaussian measure $\gamma_{\Delta}^{c}$ on $\mathbb{C}^{n} \equiv R^{2 n}$ with moments

$$
\left\langle z_{j}\right\rangle_{\gamma_{\Delta}^{c}}=0, \quad\left\langle z_{j} z_{k}\right\rangle_{\gamma_{\Delta}^{c}}=0, \quad\left\langle z_{j} \bar{z}_{k}\right\rangle_{\gamma_{\Delta}^{c}}=\Delta_{j k}, \quad 1 \leq j, k \leq n
$$

where $\Delta=\left(\Delta_{j k}\right)$ is an $n \times n$ positive semi-definite Hermitian matrix; i.e. $\gamma_{\Delta}^{c}=$ $\gamma_{\frac{1}{2} \Delta^{c}}$, where $\Delta^{c}$ is the $2 n \times 2 n$ real symmetric matrix of the inner product on $R^{2 n}$ induced by $\Delta$. As we are interested here in complex Gaussians, we drop the ' $c$ ' and write $\gamma_{\Delta}^{c}=\gamma_{\Delta}$. In particular, if $\Delta$ is a strictly positive Hermitian matrix, then $\gamma_{\Delta}$ is given by (2).

The push-forward of a Gaussian measure by a surjective linear map is also Gaussian. In the next section, we shall push forward Gaussian measures on the spaces $H_{J}^{0}\left(M, L^{N}\right)$ by linear maps that are sometimes not surjective. Since these nonsurjective push-forwards are singular measures, we need to consider the case where $\Delta$ is positive semi-definite. In this case, we use (9) to define a measure $\gamma_{\Delta}$, which we call a generalized Gaussian. If $\Delta$ has null eigenvalues, then the generalized Gaussian $\gamma_{\Delta}$ is a Gaussian measure on the subspace $\Lambda_{+} \subset \mathbb{R}^{n}$ spanned by the positive eigenvectors. (Precisely, $\gamma_{\Delta}=\iota_{*} \gamma_{\Delta \mid \Lambda_{+}}$, where $\iota: \Lambda_{+} \hookrightarrow \mathbb{R}^{n}$ is the inclusion. For the completely degenerate case $\Delta=0$, we have $\gamma_{\Delta}=\delta_{0}$.) Of course, (8) also holds for semi-positive $\Delta$. One useful property of generalized Gaussians is that the push-forward by a (not necessarily surjective) linear map $T: \mathbb{R}^{n} \rightarrow \mathbb{R}^{m}$ of a generalized Gaussian $\gamma_{\Delta}$ on $\mathbb{R}^{n}$ is a generalized Gaussian on $\mathbb{R}^{m}$ :

$$
T_{*} \gamma_{\Delta}=\gamma_{T \Delta T^{*}}
$$

Another useful property of generalized Gaussians is the following fact:

Lemma 3.1. The map $\Delta \mapsto \gamma_{\Delta}$ is a continuous map from the positive semi-definite matrices to the space of positive measures on $\mathbb{R}^{n}$ (with the weak topology).

Proof. Suppose that $\Delta^{N} \rightarrow \Delta^{0}$. We must show that $\left(\Delta^{N}, \varphi\right) \rightarrow\left(\Delta^{0}, \varphi\right)$ for a compactly supported test function $\varphi$. We can assume that $\varphi$ is $\mathcal{C}^{\infty}$. It then follows from (9) that

$$
\left(\gamma_{\Delta^{N}}, \varphi\right)=\left(\widehat{\gamma_{\Delta^{N}}}, \widehat{\varphi}\right) \rightarrow\left(\widehat{\gamma_{\Delta^{0}}}, \widehat{\varphi}\right)=\left(\gamma_{\Delta^{0}}, \varphi\right)
$$

We shall use the following 'generalized Poincaré-Borel lemma' relating spherical measures to Gaussian measures in our proof of Theorem 1.1 on asymptotics of the joint probability distributions for $S H_{J}^{0}\left(M, L^{N}\right)$.

Lemma 3.2. Let $T_{N}: \mathbb{R}^{d_{N}} \rightarrow \mathbb{R}^{k}, N=1,2, \ldots$, be a sequence of linear maps, where $d_{N} \rightarrow \infty$. Suppose that $\frac{1}{d_{N}} T_{N} T_{N}^{*} \rightarrow \Delta$. Then $T_{N *} \nu_{d_{N}} \rightarrow \gamma_{\Delta}$. 
Proof. Let $V_{N}$ be a $k$-dimensional subspace of $\mathbb{R}^{d_{N}}$ such that $V_{N}^{\perp} \subset \operatorname{ker} T^{N}$, and let $p_{N}: \mathbb{R}^{d_{N}} \rightarrow V_{N}$ denote the orthogonal projection. We decompose $T_{N}=B_{N} \circ A_{N}$, where $A_{N}=d_{N}^{1 / 2} p_{N}: \mathbb{R}^{d_{N}} \rightarrow V_{N}$, and $B_{N}=\left.d_{N}^{-1 / 2} T_{N}\right|_{V_{N}}: V_{N} \stackrel{\approx}{\rightarrow} \mathbb{R}^{k}$. Write

$$
A_{N *} \nu_{d_{N}}=\alpha_{N}, \quad T_{N *} \nu_{d_{N}}=B_{N *} \alpha_{N}=\beta_{N} .
$$

We easily see that (abbreviating $d=d_{N}$ )

$$
\alpha_{N}=A_{N *} \nu_{d}=\psi_{d} d x, \quad \psi_{d}=\left\{\begin{array}{cl}
\frac{\sigma_{d-k}}{\sigma_{d} d^{k}}\left[1-\frac{1}{d}|x|^{2}\right]^{(d-k-2) / 2} & \text { for }|x|<\sqrt{d}, \\
0 & \text { otherwise },
\end{array}\right.
$$

where $d x$ denotes Lebesgue measure on $V_{N}$, and $\sigma_{n}=\operatorname{vol}\left(S^{n-1}\right)=\frac{2 \pi^{n / 2}}{\Gamma(n / 2)}$. (The case $k=1, d=3$ of (11) is Archimedes' formula [Arc].) Since $\left[1-|x|^{2} / d\right]^{(d-k-2) / 2} \rightarrow$ $e^{-|x|^{2} / 2}$ uniformly on compacta and $\frac{\sigma_{d-k}}{\sigma_{d} d^{k}} \rightarrow \frac{1}{(2 \pi)^{k / 2}}$, we conclude that $\alpha_{N} \rightarrow \gamma_{k}$. (This is the Poincaré-Borel theorem; see Corollary 3.3 below.) Furthermore,

$$
\left(1-\frac{1}{d}|x|^{2}\right)^{(d-k-2) / 2} \leq \exp \left(-\frac{d-k-2}{2 d}|x|^{2}\right) \leq e^{\frac{k+2}{2}} e^{-\frac{1}{2}|x|^{2}}
$$

for $d \geq k+2,|x| \leq \sqrt{d}$, and hence

$$
\psi_{d_{N}}(x) \leq C_{k} e^{-|x|^{2} / 2} .
$$

Now let $\varphi$ be a compactly supported continuous test function on $\mathbb{R}^{k}$. We must show that

$$
\int \varphi d \beta_{N} \rightarrow \int \varphi d \gamma_{\Delta}
$$

Suppose on the contrary that (13) does not hold. After passing to a subsequence, we may assume that $\int \varphi d \beta_{N} \rightarrow c \neq \int \varphi d \gamma_{\Delta}$. Since the eigenvalues of $B_{N}$ are bounded, we can assume (again taking a subsequence) that $B_{N} \rightarrow B_{0}$, where

$$
B_{0} B_{0}^{*}=\lim _{N \rightarrow \infty} B_{N} B_{N}^{*}=\lim _{N \rightarrow \infty} \frac{1}{d_{N}} T_{N} T_{N}^{*}=\Delta .
$$

Hence,

$$
\begin{aligned}
\int_{\mathbb{R}^{k}} \varphi d \beta_{N}=\int_{V_{N}} \varphi\left(B_{N} x\right) \psi_{d_{N}}(x) d x & \\
& \rightarrow \int_{V_{N}} \varphi\left(B_{0} x\right) \frac{e^{-|x|^{2} / 2}}{(2 \pi)^{k / 2}} d x=\int_{V_{N}} \varphi\left(B_{0} x\right) d \gamma_{k}(x),
\end{aligned}
$$

where the limit holds by dominated convergence, using (12). By (10), we have $B_{0 *} \gamma_{k}=\gamma_{B_{0} B_{0}^{*}}=\gamma_{\Delta}$, and hence

$$
\int_{V_{N}} \varphi\left(B_{0} x\right) d \gamma_{k}(x)=\int_{\mathbb{R}^{k}} \varphi d \gamma_{\Delta} .
$$

Thus (13) holds for the subsequence, giving a contradiction. 
We note that the above proof began by establishing the Poincaré-Borel theorem (which is a special case of Lemma [3.2):

Corollary 3.3 (Poincaré-Borel). Let $P_{d}: \mathbb{R}^{d} \rightarrow \mathbb{R}^{k}$ be given by

$$
P_{d}(x)=\sqrt{d}\left(x_{1}, \ldots, x_{k}\right)
$$

Then $P_{d *} \nu_{d} \rightarrow \gamma_{k}$

\section{Proof of Theorem 1.1}

We return to our complex Hermitian line bundle $(L, h)$ on a compact almost complex $2 m$-dimensional symplectic manifold $M$ with symplectic form $\omega=\frac{i}{2} \Theta_{L}$, where $\Theta_{L}$ is the curvature of $L$ with respect to a connection $\nabla$. We now describe the $n$-point joint distribution arising from our probability space $\left(S H_{J}^{0}\left(M, L^{N}\right), \nu_{N}\right)$. We introduce the Hermitian inner product on $H_{J}^{0}\left(M, L^{N}\right)$ :

$$
\left\langle s_{1}, s_{2}\right\rangle=\int_{M} h^{N}\left(s_{1}, s_{2}\right) \frac{1}{m !} \omega^{m} \quad\left(s_{1}, s_{2} \in H_{J}^{0}\left(M, L^{N}\right)\right),
$$

and we write $\|s\|_{2}=\langle s, s\rangle^{1 / 2}$. Recall that $S H_{J}^{0}\left(M, L^{N}\right)$ denotes the unit sphere $\{\|s\|=1\}$ in $H_{J}^{0}\left(M, L^{N}\right)$ and $\nu_{N}$ denotes its Haar probability measure.

We let $J^{1}\left(M, L^{N}\right)$ denote the space of 1-jets of sections of $L^{N}$. Recall that we have the exact sequence of vector bundles

$$
0 \rightarrow T_{M}^{*} \otimes L^{N} \stackrel{\iota}{\rightarrow} J^{1}\left(M, L^{N}\right) \stackrel{\rho}{\rightarrow} L^{N} \rightarrow 0
$$

We consider the jet maps

$$
J_{z}^{1}: H_{J}^{0}\left(M, L^{N}\right) \rightarrow J^{1}(M, V)_{z}, \quad J_{z}^{1} s=\text { the } 1 \text {-jet of } s \text { at } z, \quad \text { for } z \in M .
$$

The covariant derivative $\nabla: J^{1}\left(M, L^{N}\right) \rightarrow T_{M}^{*} \otimes L^{N}$ provides a splitting of (15) and an isomorphism

$$
(\rho, \nabla): J^{1}\left(M, L^{N}\right) \stackrel{\approx}{\longrightarrow} L^{N} \oplus\left(T_{M}^{*} \otimes L^{N}\right) .
$$

Definition. The $n$-point joint probability distribution at points $P^{1}, \ldots, P^{n}$ of $M$ is the probability measure

$$
\mathbf{D}_{\left(P^{1}, \ldots, P^{n}\right)}^{N}:=\left(J_{P^{1}}^{1} \oplus \cdots \oplus J_{P^{n}}^{1}\right)_{*} \nu_{N}
$$

on the space $J^{1}\left(M, L^{N}\right)_{P^{1}} \oplus \cdots \oplus J^{1}\left(M, L^{N}\right)_{P^{n}}$.

Since we are interested in the scaling limit of $\mathbf{D}^{N}$, we need to describe this measure more explicitly: Suppose that $P^{1}, \ldots, P^{n}$ lie in a coordinate neighborhood of a point $P_{0} \in M$, and choose preferred coordinates $\left(z_{1}, \ldots, z_{m}\right)$ at $P_{0}$. We let $z_{1}^{p}, \ldots, z_{m}^{p}$ denote the coordinates of the point $P^{p}(1 \leq p \leq n)$, and we write $z^{p}=\left(z_{1}^{p}, \ldots, z_{m}^{p}\right)$. (The coordinates of $P_{0}$ are 0 .) We consider the $n(2 m+1)$ complex-valued random variables $x^{p}, \xi_{q}^{p}(1 \leq p \leq n, 1 \leq q \leq 2 m)$ on $S \mathcal{H}_{N}^{2}(X) \equiv$ $S H_{J}^{0}\left(M, L^{N}\right)$ given by

$$
\begin{gathered}
x^{p}(s)=s\left(z^{p}, 0\right) \\
\xi_{q}^{p}(s)=\frac{1}{\sqrt{N}} \frac{\partial^{h} s}{\partial z_{q}}\left(z^{p}\right), \quad \xi_{m+q}^{p}(s)=\frac{1}{\sqrt{N}} \frac{\partial^{h} s}{\partial \bar{z}_{q}}\left(z^{p}\right) \quad(1 \leq q \leq m),
\end{gathered}
$$

for $s \in S H_{J}^{0}\left(M, L^{N}\right)$. 
We now write

$$
x=\left(x^{1}, \ldots, x^{p}\right), \quad \xi=\left(\xi_{q}^{p}\right)_{1 \leq p \leq n, 1 \leq q \leq 2 m}, \quad z=\left(z^{1}, \ldots, z^{n}\right) .
$$

Using (16) and the variables $x^{p}, \xi_{q}^{p}$ to make the identification

$$
J^{1}\left(M, L^{N}\right)_{P^{1}} \oplus \cdots \oplus J^{1}\left(M, L^{N}\right)_{P^{n}} \equiv \mathbb{C}^{n(2 m+1)},
$$

we can write

$$
\mathbf{D}_{z}^{N}=D^{N}(x, \xi ; z) d x d \xi
$$

where $d x d \xi$ denotes Lebesgue measure on $\mathbb{C}^{n(2 m+1)}$.

Before proving Theorem 1.1 on the scaling limit of $\mathbf{D}_{z}^{N}$, we state and prove a corresponding result replacing $\left(S H_{J}^{0}\left(M, L^{N}\right), \nu_{N}\right)$ with the essentially equivalent Gaussian space $H_{J}^{0}\left(M, L^{N}\right)$ with the normalized standard Gaussian measure

$$
\mu_{N}:=\left(\frac{d_{N}}{\pi}\right)^{d_{N}} e^{-d_{N}|c|^{2}} d c, \quad s=\sum_{j=1}^{d_{N}} c_{j} S_{j}^{N},
$$

where $\left\{S_{j}^{N}\right\}$ is an orthonormal basis for $H_{J}^{0}\left(M, L^{N}\right)$. This measure is characterized by the property that the $2 d_{N}$ real variables $\Re c_{j}, \Im c_{j}\left(j=1, \ldots, d_{N}\right)$ are independent Gaussian random variables with mean 0 and variance $1 / 2 d_{N}$; i.e.,

$$
\left\langle c_{j}\right\rangle_{\mu_{N}}=0, \quad\left\langle c_{j} c_{k}\right\rangle_{\mu_{N}}=0, \quad\left\langle c_{j} \bar{c}_{k}\right\rangle_{\mu_{N}}=\frac{1}{d_{N}} \delta_{j k} .
$$

Our normalization guarantees that the variance of $\|s\|_{2}$ is unity:

$$
\left\langle\|s\|_{2}^{2}\right\rangle_{\mu_{N}}=1 .
$$

We then consider the Gaussian joint probability distribution

$$
\widetilde{\mathbf{D}}_{\left(P^{1}, \ldots, P^{n}\right)}^{N}=\widetilde{D}^{N}(x, \xi ; z) d x d \xi=\left(J_{P^{1}}^{1} \oplus \cdots \oplus J_{P^{n}}^{1}\right)_{*} \mu_{N} .
$$

Since $\mu_{N}$ is Gaussian and the map $J_{P^{1}}^{1} \oplus \cdots \oplus J_{P^{n}}^{1}$ is linear, it follows that the joint probability distribution is a generalized Gaussian measure of the form

$$
D^{N}(x, \xi ; z) d x d \xi=\gamma_{\Delta^{N}(z)} .
$$

We shall see below that the covariance matrix $\Delta^{N}(z)$ is given in terms of the Szegö kernel.

We have the following alternate form of Theorem 1.1

Theorem 4.1. Under the hypotheses and notation of Theorem 1.1, we have

$$
\widetilde{\mathbf{D}}_{\left(z^{1} / \sqrt{N}, \ldots, z^{n} / \sqrt{N}\right)}^{N} \longrightarrow \mathbf{D}_{\left(z^{1}, \ldots, n^{n}\right)}^{\infty} .
$$

Proof. We use the coordinates $\left(z_{1}, \ldots, z_{m}, \theta\right)$ on $X$ given by preferred coordinates at $P_{0} \in M$ and a local frame $e_{L}$ for $L$ with $\left\|e_{L}\right\|_{P_{0}}=1$ and $\left.\nabla e_{L}\right|_{P_{0}}=0$ as in $₫ 2$. The covariance matrix $\Delta^{N}(z)$ in (24) is a positive semi-definite $n(2 m+1) \times n(2 m+1)$ Hermitian matrix. If the map $J_{z^{1}}^{1} \oplus \cdots \oplus J_{z^{n}}^{1}$ is surjective, then $\Delta^{N}(z)$ is strictly positive definite and $\widetilde{D}^{N}(x, \xi ; z)$ is a smooth function. On the other hand, if the map is not surjective, then $\widetilde{D}^{N}(x, \xi ; z)$ is a distribution supported on a linear subspace. For example, in the integrable holomorphic case, $\widetilde{D}^{N}(x, \xi ; z)$ is supported on the holomorphic jets, as follows from the discussion below. 
By (8), we have

$$
\begin{gathered}
\Delta^{N}(z)=\left(\begin{array}{cc}
A & B \\
B^{*} & C
\end{array}\right), \\
(25) A=\left(A_{p^{\prime}}^{p}\right)=\left\langle x^{p} \bar{x}^{p^{\prime}}\right\rangle_{\mu_{N}}, \quad B=\left(B_{p^{\prime} q^{\prime}}^{p}\right)=\left\langle x^{p} \bar{\xi}_{q^{\prime}}^{p^{\prime}}\right\rangle_{\mu_{N}}, \quad C=\left(C_{p^{\prime} q^{\prime}}^{p q}\right)=\left\langle\xi_{q}^{p} \bar{\xi}_{q^{\prime}}^{p^{\prime}}\right\rangle_{\mu_{N}}, \\
p, p^{\prime}=1, \ldots, n, \quad q, q^{\prime}=1, \ldots, 2 m .
\end{gathered}
$$

(We note that $A, B, C$ are $n \times n, n \times 2 m n, 2 m n \times 2 m n$ matrices, respectively; $p, q$ index the rows, and $p^{\prime}, q^{\prime}$ index the columns.)

We now describe the the entries of the matrix $\Delta^{N}$ in terms of the Szegö kernel. We have by 22) and (25), writing $s=\sum_{j=1}^{d_{N}} c_{j} S_{j}^{N}$,

$$
A_{p^{\prime}}^{p}=\left\langle x^{p} \bar{x}^{p^{\prime}}\right\rangle_{\mu_{N}}=\sum_{j, k=1}^{d_{N}}\left\langle c_{j} \bar{c}_{k}\right\rangle_{\mu_{N}} S_{j}^{N}\left(z^{p}, 0\right) \overline{S_{k}^{N}\left(z^{p^{\prime}}, 0\right)}=\frac{1}{d_{N}} \Pi_{N}\left(z^{p}, 0 ; z^{p^{\prime}}, 0\right) .
$$

We need some more notation to describe the matrices $B$ and $C$. Write

$$
\nabla_{q}=\frac{1}{\sqrt{N}} \frac{\partial^{h}}{\partial z_{q}}, \quad \nabla_{m+q}=\frac{1}{\sqrt{N}} \frac{\partial^{h}}{\partial \bar{z}_{q}}, \quad 1 \leq q \leq m .
$$

We let $\nabla_{q}^{1}$, resp. $\nabla_{q}^{2}$, denote the differential operator on $X \times X$ given by applying $\nabla_{q}$ to the first, resp. second, factor $(1 \leq q \leq 2 m)$. By differentiating (26), we obtain

$$
\begin{aligned}
B_{p^{\prime} q^{\prime}}^{p} & =\frac{1}{d_{N}} \bar{\nabla}_{q^{\prime}}^{2} \Pi_{N}\left(z^{p}, 0 ; z^{p^{\prime}}, 0\right), \\
C_{p^{\prime} q^{\prime}}^{p q} & =\frac{1}{d_{N}} \nabla_{q}^{1} \bar{\nabla}_{q^{\prime}}^{2} \Pi_{N}\left(z^{p}, 0 ; z^{p^{\prime}}, 0\right) .
\end{aligned}
$$

We now use the scaling asymptotics of the almost holomorphic Szegö kernel $\Pi_{N}(x, y)$ given in [ShZe2]. In addition to the above assumption on the local frame $e_{L}$, we further assume that

$$
\left.\nabla^{2} e_{L}\right|_{P_{0}}=-\left.(g+i \omega) \otimes e_{L}\right|_{P_{0}} \in T_{M}^{*} \otimes T_{M}^{*} \otimes L .
$$

(In [ShZe3], we called such an $e_{L}$ a 'preferred frame' at $P_{0}$, and the resulting coordinates were called 'Heisenberg coordinates.') Then we have (see ShZe3, Theorem 2.3)

$$
\begin{aligned}
N^{-m} & \Pi_{N}\left(\frac{u}{\sqrt{N}}, \frac{\theta}{N} ; \frac{v}{\sqrt{N}}, \frac{\varphi}{N}\right) \\
= & \frac{1}{\pi^{m}} e^{i(\theta-\varphi)+u \cdot \bar{v}-\frac{1}{2}\left(|u|^{2}+|v|^{2}\right)} \\
\quad & \times\left[1+\sum_{r=1}^{K} N^{-\frac{r}{2}} b_{r}\left(P_{0}, u, v\right)+N^{-\frac{K+1}{2}} R_{K}\left(P_{0}, u, v, N\right)\right],
\end{aligned}
$$

where $\left\|R_{K}\left(P_{0}, u, v, N\right)\right\|_{\mathcal{C}^{j}(\{|u|+|v| \leq \rho\})} \leq C_{K, j, \rho}$ for $j=1,2,3, \ldots$

It follows from (26) $-(28)$ and (29), recalling (16) $-(7)$, that

$$
\Delta^{N}\left(\frac{z}{\sqrt{N}}\right) \rightarrow \Delta^{\infty}(z)=\frac{m !}{c_{1}(L)^{m}}\left(\begin{array}{cc}
A^{\infty}(z) & B^{\infty}(z) \\
B^{\infty}(z)^{*} & C^{\infty}(z)
\end{array}\right)
$$

in the notation of (4). 
Finally, we apply Lemma 3.1 to (24) and conclude that

$$
\widetilde{\mathbf{D}}_{z / \sqrt{N}}^{N}=\gamma_{\Delta^{N}(z / \sqrt{N})} \rightarrow \gamma_{\Delta^{\infty}(z)}=\mathbf{D}_{z}^{\infty}
$$

Proof of Theorem 1.1. The proof is similar to that of Theorem 4.1 This time we define

$$
\Delta^{N}=\frac{1}{d_{N}} \mathcal{J}_{N} \mathcal{J}_{N}^{*}: H^{0}\left(M, L^{N}\right) \rightarrow \mathbb{C}^{n(2 m+1)},
$$

where $\mathcal{J}_{N}=J_{P^{1}}^{1} \oplus \cdots \oplus J_{P^{n}}^{1}$ under the identification (20). We see immediately that $\Delta^{N}$ is given by (26) $-(28)$, and the conclusion follows from Lemma 3.2 and (44).

Remark. There are other similar ways to define the joint probability distribution that have the same universal scaling limits. One of these is to use the (unnormalized) standard Gaussian measure $\gamma_{2 d_{N}}$ on $H_{J}^{0}\left(M, L^{N}\right)$ in place of the normalized Gaussian $\mu_{N}$ in Theorem 4.1 to obtain joint densities

$$
D_{\#}^{N}(x, \xi ; z)=D^{N}\left(\frac{x}{N^{m / 2}}, \frac{\xi}{N^{m / 2}} ; z\right) .
$$

Then we would have instead

$$
D_{\#}^{N}\left(N^{m / 2} x, N^{m / 2} \xi ; N^{-1 / 2} z\right) d x d \xi \rightarrow \gamma_{\Delta \infty}(z) .
$$

Another similar result is to let $\lambda_{N}$ denote normalized Lebesgue measure on the unit ball $\{\|s\| \leq 1\}$ in $H_{J}^{0}\left(M, L^{N}\right)$ and to let $\widehat{\mathbf{D}}_{z}^{N}=\mathcal{J}_{N *} \lambda_{N}$. By a similar argument as above, we also have $\widehat{\mathbf{D}}_{z / \sqrt{N}}^{N} \rightarrow \gamma_{\Delta^{\infty}(z)}$.

\section{REFERENCES}

[Arc] Archimedes, On the Sphere and Cylinder (Greek), Syracuse, ca. 257BC.

[Aur] Denis Auroux, Estimated transversality in symplectic geometry and projective maps, to appear in Proc. International KIAS Conference (Seoul, 2000), http://xxx.lanl.gov/abs/math.SG/0010052.

[BSZ1] P. Bleher, B. Shiffman and S. Zelditch, Universality and scaling of correlations between zeros on complex manifolds, Invent. Math. 142 (2000), 351-395.

[BSZ2] P. Bleher, B. Shiffman and S. Zelditch, Universality and scaling of zeros on symplectic manifolds, in Random Matrix Models and Their Applications, P. Bleher and A. Its (Eds.), MSRI Publications 40, Cambridge Univ. Press, 2001, pp. 31-69.

[BSZ3] P. Bleher, B. Shiffman and S. Zelditch, Correlations between zeros and supersymmetry, Commun. Math. Phys. 224 (2001), 255-269.

[BoGu] L. Boutet de Monvel and V. Guillemin, The Spectral Theory of Toeplitz Operators, Ann. Math. Studies 99, Princeton Univ. Press, Princeton, 1981. MR 85j:58141

[BoSj] L. Boutet de Monvel and J. Sjöstrand, Sur la singularité des noyaux de Bergman et de Szegö, Asterisque 34-35 (1976), 123-164. MR 58:28684

[Don] S. K. Donaldson, Symplectic submanifolds and almost complex geometry, J. Diff. Geom. 44 (1996), 666-705. MR 98h:53045]

[ShZe1] B. Shiffman and S. Zelditch, Random almost holomorphic sections of ample line bundles on symplectic manifolds, (preprint 2000), http://xxx.lanl.gov/abs/math.SG/0001102.

[ShZe2] B. Shiffman and S. Zelditch, Asymptotics of almost holomorphic sections of ample line bundles on symplectic manifolds, J. Reine Angew. Math. 544 (2002), 181-222. 
[ShZe3] B. Shiffman and S. Zelditch, Random polynomials and Levy concentration of measure, (in preparation).

[Woo] N. M. J. Woodhouse, Geometric Quantization, Clarendon Press, Oxford, 1992. MR 94a:58082

Department of Mathematics, Johns Hopkins University, Baltimore, Maryland 21218

E-mail address: shiffman@math.jhu.edu

Department of Mathematics, Johns Hopkins University, Baltimore, Maryland 21218

E-mail address: zelditch@math.jhu.edu 